\title{
Integrated Pharmacies at Community Mental Health Centers: Medication Adherence and Outcomes
}

\author{
W. Abel Wright, MS; Jack M. Gorman, MD; Melissa Odorzynski, PharmD, MPH; \\ Mark J. Peterson, RPh; and Carol Clayton, PhD
}

\begin{abstract}
BACKGROUND: Patients receiving psychiatric services at community mental health centers (CMHCs) are often prescribed medication that is critical to the treatment of behavioral health conditions, including schizophrenia, bipolar disorder, anxiety, and depression. Previous studies have shown correlation between rates of medication adherence and risk of hospitalization, but potential differences in medication adherence and other outcomes for patients of CMHCs by pharmacy type have not been widely studied.
\end{abstract}

OBJECTIVE: To determine potential benefits of placing a pharmacy within a mental health service delivery setting on both adherence to medication and health outcomes.

METHODS: A retrospective cohort analysis of medication adherence rates, hospital and emergency department (ED) use, and related costs between patients of CMHCs was conducted using integrated pharmacies versus community pharmacies. Data were from Medicaid claims paid by Southwest Michigan Behavioral Health for all (behavioral and nonbehavioral) inpatient and outpatient services as well as pharmacy prescriptions filled from April 1, 2014, through April 30, 2015. The primary study analysis was composed of an adult dataset representing persons served from 1 of the 2 CMHCs who had filled at least 2 prescriptions for a specific medication from 1 of 2 Genoa pharmacies located in a CMHC during the study period. Each unique patient dataset in the treatment group was matched to a corresponding control patient dataset prescribed the same medication using a modified version of the Gale-Shapley algorithm. The primary analysis compared medication possession ratio, which is a measure of adherence that indicates gaps or oversupply in a patient's medication use history. Statistical tests were performed using the R statistical programming language and Microsoft Excel.

RESULTS: Patients using pharmacies integrated within the CMHCs had higher medication adherence rates, lower rates of hospitalization, and lower ED use than those filling their prescriptions at community pharmacies. These results were associated with a cost savings of $\$ 58$ per member per month (approximately $\$ 700,000$ per 1,000 patients annually).

CONCLUSIONS: Pharmacies integrated within CMHCs not only can improve medication adherence but also can reduce the need for other expensive health care services.

J Manag Care Spec Pharm. 2016;22(11):1330-36

Copyright $\odot 2016$, Academy of Managed Care Pharmacy. All rights reserved.

\section{What is already known about this subject}

Poor adherence to medication is a major problem across all disease categories, including behavioral health diagnoses, and a leading cost driver for health plans and payers.

There is considerable industry-focused attention driving ways to address this problem, including performance-based metrics. One reason for poor medication adherence is physical barriers to obtaining medication.

\section{What this study adds}

Locating integrated pharmacies on-site at community mental health centers improves medication adherence and health outcomes.

Improvements in health outcomes such as lower hospitalization rates and lower emergency department utilization translate into lower overall costs.

$\mathrm{P}$ oor adherence to prescribed medication is widely recognized as a major determinant of poor outcomes across all disease categories. ${ }^{1,2}$ Patients with behavioral health diagnoses are particularly prone to nonadherence to psychiatric and other medication classes. ${ }^{3-7}$ Nonadherence to medication has been linked to increased use of health care services, including increased hospitalizations and use of emergency department (ED) services. ${ }^{8-11}$ Thus, nonadherence to prescribed medication is a risk factor for worsening illness status, diminished function, and increased health care expenditures.

The reasons that patients do not take medications as prescribed include denial of illness, cost of medication, relationship with prescribers, physical barriers to obtaining medication, comorbid substance use, and adverse side effects, among others (e.g., complexity of the medication regimen). 2,12-15 Pharmacies that operate on-site within clinics may help reduce these barriers, primarily by addressing the physical barriers to obtaining medication, but potentially via other means, such as embedding a pharmacist into the clinical care team. It should be noted that in this study the physical location of the pharmacy, whether in or out of the clinic, is the primary distinguishing characteristic between pharmacies compared, but other factors may affect differences in adherence rates and outcomes, such as services offered and interventions performed.

In this analysis, we examined the potential benefits of placing a pharmacy within a mental health service delivery setting on both adherence to medication and health outcomes. We compared these outcomes between patients who filled their prescriptions at the pharmacy located within the community mental health center (CMHC) in which they received care and those who filled their prescriptions at pharmacies outside of their CMHC. We hypothesized that the former group would demonstrate significantly better adherence to prescribed 


\begin{tabular}{|c|c|c|c|c|}
\hline Control & $\begin{array}{c}\text { Genoa } \\
\text { (Treatment) }\end{array}$ & $\begin{array}{c}\text { Net } \\
\text { Difference }\end{array}$ & $\begin{array}{c}\% \\
\text { Difference } \\
\text { (Absolute } \\
\text { Value) }\end{array}$ & $P$ Value ${ }^{a}$ \\
\hline 1,378 & 1,378 & & & \\
\hline 45.1 & 45.3 & 0.2 & 1 & 0.003 \\
\hline 52 & 52 & 0 & 0 & 1.000 \\
\hline 7 & 7 & 0 & 0 & 1.000 \\
\hline 0 & 0 & 0 & 0 & N/A \\
\hline 1 & 1 & 0 & 0 & 1.000 \\
\hline 1 & 1 & 0 & 0 & 1.000 \\
\hline 1 & 1 & 0 & 0 & 1.000 \\
\hline 1 & 1 & 0 & 0 & 1.000 \\
\hline 5 & 5 & 0 & 0 & 1.000 \\
\hline 0 & 0 & 0 & 0 & 1.000 \\
\hline 1 & 1 & 0 & 0 & 1.000 \\
\hline 0 & 0 & 0 & 0 & N/A \\
\hline 6 & 6 & 0 & 0 & 1.000 \\
\hline 0.28 & 0.30 & 0.02 & 6 & 0.150 \\
\hline 0.96 & 0.98 & 0.03 & 3 & 0.008 \\
\hline 1.12 & 1.16 & 0.04 & 4 & 0.015 \\
\hline 0.0007 & 0.0007 & 0.00 & 0 & N/A \\
\hline 0.0022 & 0.0022 & 0.00 & 0 & N/A \\
\hline 0.0000 & 0.0000 & 0.00 & 0 & N/A \\
\hline 0.0000 & 0.0000 & 0.00 & 0 & N/A \\
\hline 0.0007 & 0.0007 & 0.00 & 0 & N/A \\
\hline 0.0022 & 0.0022 & 0.00 & 0 & N/A \\
\hline 0.0261 & 0.0261 & 0.00 & 0 & N/A \\
\hline 0.0000 & 0.0000 & 0.00 & 0 & N/A \\
\hline 0.0261 & 0.0261 & 0.00 & 0 & N/A \\
\hline 0.21 & 0.21 & 0.00 & 0 & N/A \\
\hline 0.37 & 0.37 & 0.00 & 0 & N/A \\
\hline
\end{tabular}

Patient-medication pairs, $n$

Age (years)

Female, \%

Proportion of population with diagnosis for serious mental illness, $\%$

Proportion of population with diagnosis for ADHD, \%

Proportion of population with diagnosis for anxiety, \%

Proportion of population with diagnosis for bipolar disorder, \%

Proportion of population with diagnosis for dementia, \%

Proportion of population with diagnosis for depression, \%

Proportion of population with diagnosis for developmental disorder or intellectual disability, \%

Proportion of population with diagnosis for mood disorder, \%

Proportion of population with diagnosis for other BH conditions, \%

Proportion of population with diagnosis for PTSD, \%

Proportion of population with diagnosis for schizophrenia or psychosis, $\%$

Mean CCI score per capita

Mean CDPS score prospective per capita

Mean CDPS score concurrent per capita

Number of hospitalizations per capita

Total hospital LOS per capita

Number of hospitalizations per capita: behavioral

Hospital LOS per capita: behavioral

Number of hospitalizations per capita: nonbehavioral

Hospital LOS per capita: nonbehavioral

Number of ED visits per capita

Number of ED visits per capita: behavioral

Number of ED visits per capita: nonbehavioral

Number of behavioral medication prescribers per capita

Number of behavioral medication actives prescribed per capita

0.37

${ }^{a}$ For chi-square tests where the variable had the same value across every subject in both groups, the $P$ value cannot be computed. For the Wilcoxon signed rank test, where each pair is an exact match for the variable being evaluated, there is no difference between the paired observations, and the P value cannot be computed. "N/A" has been inserted in place of a $P$ value, indicating a perfect match between groups for that variable.

$A D H D=$ attention deficit hyperactivity disorder; $B H=$ behavioral health; $C C I=$ Charlson Comorbidity Index; CDPS=Chronic Illness and Disability Payment System;

$E D=$ emergency department; LOS = length of stay; PTSD = post-traumatic stress disorder

medications and improved health outcomes as measured by rates of hospitalization and ED use. We also examined whether patients who used the in-house pharmacies generated lower health care costs.

\section{Methods}

\section{Setting}

Genoa, a QoL Healthcare Company, is a provider of specialized pharmacy services that has partnered with more than 300 CMHCs nationally since 2000. With the goal of improving medication adherence and positively affecting patient health outcomes, Genoa has maintained pharmacies at the 2 CMHCs in this study since 2006. In-house pharmacies are located close to both the providers who are writing prescriptions and the patients who need to fill them. Genoa has implemented this integrated model under the hypothesis that it may have a positive impact on adherence and outcomes due to increased levels of communication and coordination between pharmacy and provider, as well as convenience for the patient. Patients at the CMHCs have the choice to fill their medications at the in-house pharmacies or elsewhere.

\section{Data Source}

Data for this project consisted of Medicaid claims paid by Southwest Michigan Behavioral Health (SWMBH) for all (behavioral and nonbehavioral) inpatient and outpatient services as well as pharmacy prescriptions filled. Additionally, demographic data were obtained from state Medicaid administrative patient files. All patient data were de-identified. Because exact spend data at the claims level were not provided 
per the state's contract with SWMBH, we examined potential differences in health care costs between the groups by combining differences in hospitalization/ED rates with a per-episode cost estimate for the state of Michigan of $\$ 2,086$ per inpatient day for each hospitalization and $\$ 1,233$ per ED visit. ${ }^{16,17}$ The time period for the comparative analysis was based on use from April 1, 2014, through April 30, 2015.

\section{Sample Selection}

For the primary study analysis, the study group was composed of an adult patient dataset from 1 of the 2 CMHCs that had filled at least 2 prescriptions for a specific medication from 1 of 2 Genoa pharmacies located in a CMHC during the study period. A specific medication was defined as a unique formulation specific to the generic ingredient(s), route of administration, dosage form, and strength. A matched control group was composed of adult CMHC patients who had filled at least 2 prescriptions for a specific medication from a community pharmacy outside of a CMHC. In order to be included in the analysis, the study group dataset had to include patients who had filled at least $95 \%$ of their prescriptions for the specific medication at an in-house Genoa pharmacy. The control group dataset included patients who had to have filled at least 95\% of their prescriptions for the specific medication at an outside community pharmacy.

Each unique patient dataset from the treatment group was matched to a corresponding control group patient dataset prescribed the same medication based on minimum differences across selected demographic, diagnostic, and utilization variables (see Table 1), using a modified version of the Gale-Shapley algorithm. Unique medication, sex, dual-eligible status, behavioral health diagnostic history and serious mental illness status, prior history of behavioral medication use, and prior hospital/ED use were matched exactly. After identifying potential matches based on the above attributes, we applied a second screening criterion such that a control group patient dataset needed to be within 1 standard deviation above or below the matched treatment patient dataset on values for age, overall illness severity, and first prescription fill date during the study period. Goodness-of-fit was evaluated by testing for statistically significant differences between the 2 groups across the preselected matching variables. Among these matching variables were 3 measures of overall illness severity: the Charlson Comorbidity Index (CCI) and both the Prospective and the Concurrent scales of the Chronic Illness and Disability Payment System (CDPS). ${ }^{18,19}$

\section{Adherence and Outcomes Measurements}

The primary analysis compared medication possession ratio (MPR), which is a measure of adherence that indicates gaps or oversupply in a patient's medication use history. MPR was calculated as of the end of the study period on all filled dates and days supply within a 1-year period ending 90 days before the end of the study period, for each unique medication.

\section{MPR = (total days supply minus last supply) divided by} (number of days from first fill date to last)

Given that MPR is specific to a given medication, the subject of analysis in this study was the patient/medication pair, and in some cases a single patient was evaluated for several medications.

A secondary analysis investigated rates of hospitalization and ED visits. Hospitalization and ED visits were identified based on inpatient and emergency revenue codes and Current Procedural Terminology, 4th Edition, codes. A single hospitalization and/or ED visit was counted once for each time an adult received inpatient or emergency care for any continuous period without a 1-day gap in inpatient or emergency care. These analyses were further broken down for behavioral and nonbehavioral hospitalizations and ED visits and for patients who were on all medications, on psychotropics, and psychotropic medication subclasses.

Using an estimated cost of $\$ 2,086$ per hospital inpatient day and $\$ 1,233$ per ED visit, we extrapolated savings to payers for adults participating in Genoa's in-house pharmacy program based on the difference between groups in hospitalization and ED rates.

\section{Statistical Analysis}

The Student's t-test statistic, single tailed, was used to compare MPR. In addition to comparing overall MPR between the groups, we separately compared MPR for several classes of medications and for dual-eligible and nondual-eligible adult datasets. The Wilcoxon sign rank test with continuity correction was used to evaluate per-member-per-month (PMPM) hospitalization and ED rates between the 2 groups for the outcomes period, with the alternative hypothesis being that the treatment group had the lesser PMPM rate. The 2-sided Wilcoxon sign rank test with continuity correction was used to compare the groups on the selected nondichotomous matching variables. Dichotomous matching variables were evaluated using the chi-square test. Statistical tests were performed using the R statistical programming language and Microsoft Excel. Excel was used for tabulating data and evaluating the $\mathrm{t}$-test and the chi-square test, and $\mathrm{R}$ was used for calculating the Wilcoxon sign rank test. Statistical significance was set at $P<0.050$ for all tests.

\section{Results}

The matching process resulted in 1,378 unique patient-medication matches across the treatment group and the control groups. Tests for significant differences between groups indicated comparability across matching variables. Although 4 of the matching variables were statistically or nearly statistically significantly different between groups, as can be seen in Table 1 , 


\begin{tabular}{|c|c|c|c|c|}
\hline \multirow[b]{2}{*}{ Exclusion Status and Reason } & \multicolumn{2}{|c|}{ Patient/Medication Regimens } & \multicolumn{2}{|c|}{ Unique Patients } \\
\hline & $\mathbf{n}$ & $\%$ & $\mathbf{n}$ & $\%$ \\
\hline Drug not recognized & 1 & 0.01 & 1 & 0.04 \\
\hline Has no baseline data to use for matching & 20 & 0.24 & 5 & 0.20 \\
\hline Not an adult patient & 600 & 7.06 & 179 & 7.13 \\
\hline Treatment group: not matched to a control subject & 4,248 & 49.95 & 899 & 35.83 \\
\hline Treatment: fewer than 2 prescriptions from in-house Genoa pharmacy & 2,257 & 26.54 & 929 & 37.03 \\
\hline Included in analysis & 1,378 & 16.20 & 496 & 19.77 \\
\hline Total & 8,504 & 100 & 2,509 & 100 \\
\hline
\end{tabular}

\section{TABLE 3 Comparison of Medication Possession Ratio Outcomes by Cohort-Medication Group}

\begin{tabular}{|c|c|c|c|c|c|}
\hline \multirow[b]{2}{*}{ Drug Category } & \multicolumn{2}{|c|}{ MPR Population Mean at End of Study } & \multicolumn{2}{|c|}{ Number } & \multirow[b]{2}{*}{$P$ Value } \\
\hline & Genoa & Control & Genoa & Control & \\
\hline All medications & 0.957 & 0.819 & 973 & 973 & $<0.001$ \\
\hline All medications: dual & 0.944 & 0.872 & 238 & 238 & $<0.001$ \\
\hline All medications: nondual & 0.962 & 0.802 & 735 & 735 & $<0.001$ \\
\hline Psychotropic & 0.983 & 0.845 & 494 & 494 & $<0.001$ \\
\hline All antipsychotics & 1.001 & 0.867 & 136 & 136 & $<0.001$ \\
\hline Atypical antipsychotics & 1.000 & 0.871 & 132 & 132 & $<0.001$ \\
\hline Benzodiazepines & 1.000 & 0.834 & 65 & 65 & $<0.001$ \\
\hline Antidepressants & 0.946 & 0.816 & 154 & 154 & $<0.001$ \\
\hline Mood stabilizers $^{\mathrm{a}}$ & 1.028 & 0.849 & 69 & 69 & $<0.001$ \\
\hline
\end{tabular}

the absolute differences in these measures (age, CCI, CDPS Score Prospective, CDPS Score Concurrent) were felt to be too small to warrant any statistical correction and introduced conservative bias, if any. Utilization variables used for matching, such as hospital/ED use, were based on a 3-month interval prior to the outcomes study period.

Some patient datasets were excluded from the study for a variety of reasons documented in Table 2. Failure to identify a close match was the leading reason these datasets were excluded from the analysis.

Analysis of adherence to medication as of the end of the study period showed that the MPR for the study group was higher than for the control group across all medication categories examined (see Table 3). These differences in MPR between the 2 groups were statistically significant in every comparison. Sample sizes for each medication category varied depending on data available and eligibility as of the end of the study period.

MPR scores were calculated as the summation of a year's data for each patient dataset. Adherence to all medications over the prior year was significantly higher for the study group as of the final month of the study period.
In addition to higher rates of adherence, lower rates of hospitalization and ED use were observed in the study group during the 13-month outcomes study period, particularly for behavioral services, among subsets of the total cohort (Table 4) by medication group.

Based on the difference in PMPM rates and an assumed cost of $\$ 18,964$ per behavioral hospitalization $(\$ 2,086$ per day for 9.09 days), approximately $\$ 57$ was saved per member-month for behavioral hospitalizations ( $\$ 56,892$ per 1,000 membermonths) for the overall combined cohort of datasets representing individuals who filled medications included in the study at an integrated pharmacy. The study group is estimated to have saved approximately $\$ 226,084$ from hospitalization rate reductions over the course of the 3,974 member-months included in the outcomes period.

Similarly, based on the difference in PMPM rates and an average cost of $\$ 1,233$ per ED visit, approximately $\$ 1.23$ was saved per member-month for behavioral ED visits ( $\$ 1,233$ per 1,000 member-months) for patients who filled any prescription at an in-house Genoa pharmacy. The study group is estimated to have saved approximately $\$ 4,900$ from ED reductions over the course of the 3,974 member-months included in the outcomes period. 
Integrated Pharmacies at Community Mental Health Centers: Medication Adherence and Outcomes

TABLE 4 Comparison of Utilization Outcomes Measures for All Medications Cohort-Medication Group ${ }^{a}$

\begin{tabular}{|c|c|c|c|c|c|c|}
\hline \multirow[b]{2}{*}{ Measure } & \multicolumn{2}{|c|}{ PMPM Rate } & \multicolumn{2}{|c|}{ Rate per 1,000 Member-Months } & \multirow[b]{2}{*}{ Risk Ratio } & \multirow[b]{2}{*}{$P$ Value ${ }^{b}$} \\
\hline & Study & Control & Study & Control & & \\
\hline Number of hospitalizations & 0.017 & 0.020 & 17 & 20 & 0.88 & 0.018 \\
\hline Number of hospitalizations: behavioral & 0.004 & 0.007 & 4 & 7 & 0.60 & 0.001 \\
\hline Number of hospitalizations: nonbehavioral & 0.013 & 0.013 & 13 & 13 & 1.03 & 0.384 \\
\hline Total hospital LOS & 0.102 & 0.151 & 102 & 151 & 0.68 & 0.022 \\
\hline Total hospital LOS: behavioral & 0.039 & 0.061 & 39 & 61 & 0.65 & 0.004 \\
\hline Total hospital LOS: nonbehavioral & 0.063 & 0.090 & 63 & 90 & 0.70 & 0.291 \\
\hline Number of ED visits & 0.082 & 0.087 & 82 & 87 & 0.95 & 0.025 \\
\hline Number of ED visits: behavioral & 0.007 & 0.008 & 7 & 8 & 0.82 & 0.006 \\
\hline Number of ED visits: nonbehavioral & 0.076 & 0.079 & 76 & 79 & 0.96 & 0.091 \\
\hline
\end{tabular}

The total savings estimate, which is based on the $19.8 \%$ of unique in-house Genoa patients whose datasets were eligible for inclusion in the analysis, is approximately $\$ 230,984$ for the 13-month period from April 1, 2014, through April 30, 2015.

We further divided the sample into medication subgroups representing only the patient datasets that included taking any psychotropic medication, any antipsychotic, any atypical (second-generation) antipsychotic, and any mood stabilizer. Analyses of hospitalization and ED visit rates were the same among each of these groups as for the entire (all medications) sample (results available on request from authors).

\section{Discussion}

Studies have identified many reasons for poor adherence to prescribed medications, including denial of illness, relationships with providers, and barriers to obtaining medication such as lack of transportation to a pharmacy. In the present study, we found that patients who accessed pharmacies that are on-site at CMHCs had better medication adherence, fewer hospitalizations and ED visits, and reduced medical care costs compared with patients who used pharmacies in the community. The finding that patients who obtain medications at the in-house pharmacy had higher MPR scores, indicating superior adherence, than those who obtained medications at community pharmacies applied not only to the MPR score calculated for all medications, but also for specific classes of psychotropic medications (Figure 1). This difference may be due to proximity: When patients do not have to make an extra trip to fill prescriptions, they may be more likely to get their medications and therefore more adherent to prescribed medication regimens.

However, there are other reasons on-site pharmacies are associated with better adherence and outcomes. When the pharmacy is integrated into the clinic setting, pharmacists have an opportunity to become part of the patient's care team and can help identify and resolve medication issues efficiently.
Services may be designed specifically to address the needs of the clinic's patients and can be tailored to address individuals' reasons for poor adherence in the behavioral health setting. These enhanced services may include specialty adherence packaging, personal refill reminders, and enhanced communication with both the patient and the providers.

Because of the large number of community pharmacies outside of the CMHCs that were used by the control group patients in this study, it was not possible for us to get information about the extent to which these pharmacies also offered such enhanced services. Therefore, it is not yet possible for us to determine the precise reasons these on-site pharmacies are associated with superior adherence and outcomes. We hope to pursue these questions in future work.

Although our analyses do not permit us to assert that improved adherence was the sole reason for the association of superior adherence with the lower number of hospitalizations and ED visits, other studies have shown a positive relationship between improved medication adherence and improved health outcomes. ${ }^{20}$ Thus, it is possible that the services provided by the integrated pharmacy were an important mediator of improved adherence, which, in turn, was a factor in reducing hospitalizations and ED visits.

As a discussion point relative to bending the cost curve, the potential cost savings due to reduced hospital and ED use for patients engaging with on-site pharmacies are worthy of note. If the estimated savings identified in the Results section are extrapolated, with standard caveats about generalizability, at the same rate to the remainder of the patients who filled prescriptions at an in-house pharmacy but were not included in the analysis, then the total savings estimate for the 13-month period would be approximately $\$ 1,168,358$ for 2,509 unique patients. While this estimate is speculative at this time-in the absence of data to support this level of generalization-it is considerable and certainly warrants further analysis. 


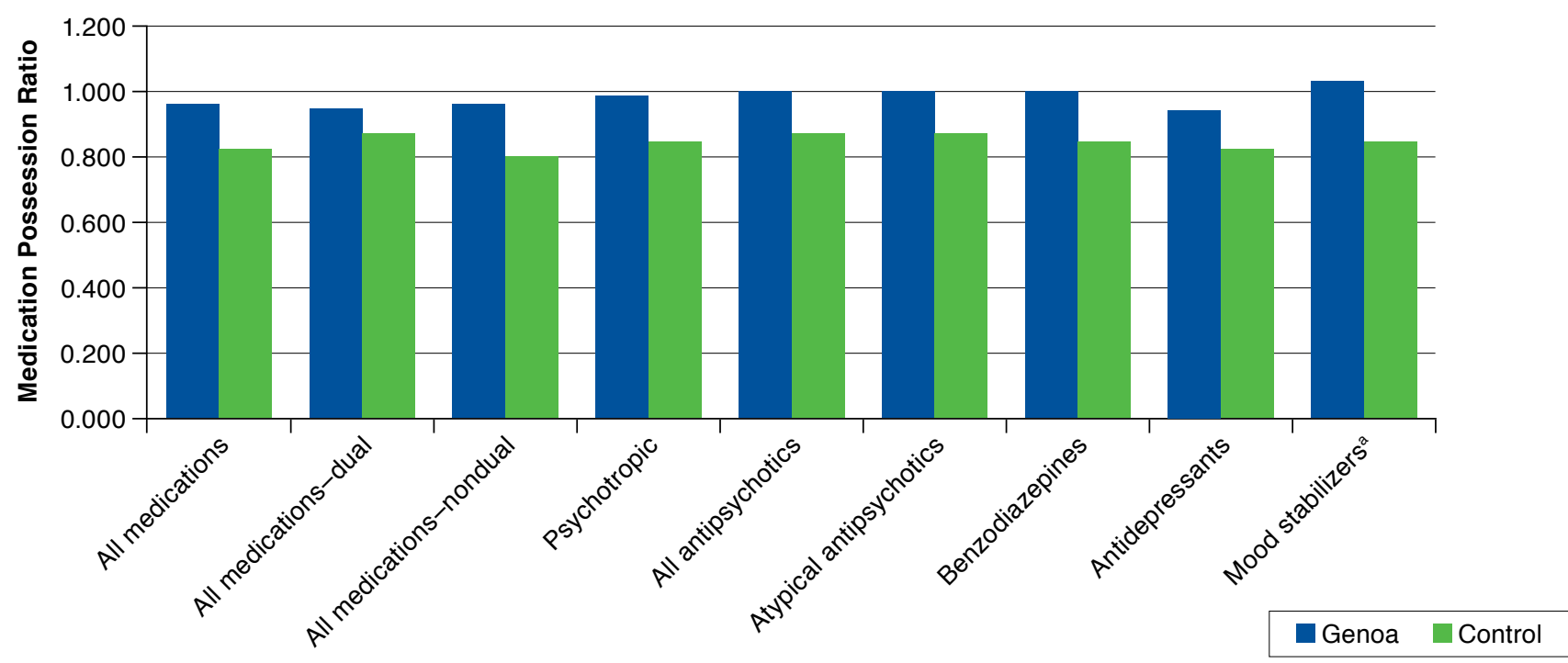

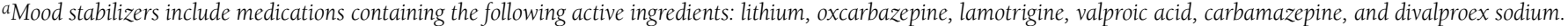

\section{Limitations}

This study is subject to several limitations. We were able to only find acceptable matches for approximately 16\% of the patient data in the study group, which limits the extent to which these results generalize to the entire patient group. As a post hoc observational study, randomized control design was not an option, and while every attempt was made to adjust for bias in the selection of the study group when selecting the matched control group, it is possible that there were additional biases that were not adjusted for and that could have affected the results of the analysis. Therefore, causal inferences cannot be made with certainty. The use of cost estimates rather than paid amounts in the cost calculations is a limitation, given the potential for differences between the source of the estimates and the study population in severity of case mix and payment arrangements. Finally, as noted above, we did not isolate differences in services offered between the study group in-house pharmacies and the control group community pharmacies and therefore do not yet know what factors beyond location may be responsible for the association with improved adherence.

\section{Conclusions}

Physical proximity to the source of prescription medication is a potentially important factor in whether a patient is adherent to a prescribed medication, and removing a physical barrier to obtaining medication may be a mediating factor for improved adherence and decreased need for other expensive health care services.

\section{Authors}

W. ABEL WRIGHT, MS; JACK M. GORMAN, MD, and CAROL CLAYTON, PhD, Care Management Technologies, Morrisville, North Carolina. MELISSA ODORZYNSKI, PharmD, MPH, and MARK J. PETERSON, RPh, Genoa, a QoL Healthcare Company, Eagan, Minnesota.

AUTHOR CORRESPONDENCE: Carol Clayton, PhD, Care Management Technologies, 808 Aviation Pkwy., Ste. 700, Morrisville, NC 27560. E-mail: cclayton@reliaslearning.com.

\section{DISCLOSURES}

Southwest Michigan Behavioral Health sponsored this study, which was funded by Genoa, a QoL Healthcare Company. SWMBH is a client of Care Management Technologies and permitted the use of its data for this analysis. Wright and Clayton are employed by Care Management Technologies, which was contracted by Genoa to conduct this analysis. Gorman owns Franklin Behavioral Health Consultants and reports consulting fees from Care Management Technologies; Gorman also reports stock ownership in various pharmaceutical companies. Odorzynski and Peterson are employed by Genoa.

Study concept and design were contributed by Clayton, Odorzynski, Peterson, and Gorman, with assistance from Wright. Wright took the lead in data collection, with assistance from Clayton and Odorzynski, and data interpretation was performed by Wright and Gorman, with assistance from Odorzynski, Clayton, and Peterson. The manuscript was written by Gorman, Wright, and Odorzynski, assisted by Clayton and Peterson, and revised by Clayton, Gorman, Odorzynski, and Peterson.

\section{ACKNOWLEDGMENTS}

The authors thank Southwest Michigan Behavioral Health for providing the de-identified dataset for this analysis. 


\section{REFERENCES}

1. Osterberg L, Blaschke T. Adherence to medication. N Engl J Med. 2005;353(5):487-97.

2. Ho PM, Bryson CL, Rumsfeld JS. Medication adherence: its importance in cardiovascular outcomes. Circulation. 2009;119(23):3028-35.

3. DiMatteo MR, Lepper HS, Croghan TW. Depression is a risk factor for noncompliance with medical treatment: meta-analysis of the effects of anxiety and depression on patient adherence. Arch Intern Med. 2000;160(14):2101-07.

4. Braithwaite RS, McGinnis KA, Conigliaro J, et al. A temporal and doseresponse association between alcohol consumption and medication adherence among veterans in care. Alcohol Clin Exp Res. 2005;29(7):1190-97.

5. Worth TE. Medication adherence strategies: we can do better. Am J Nursing. 2010;110(4):15.

6. Grenard JL, Munjas BA, Adams JL, et al. Depression and medication adherence in the treatment of chronic diseases in the United States: a metaanalysis. J Gen Intern Med. 2011;26(10):1175-82.

7. Grodensky CA, Golin CE, Ochtera RD, Turner BJ. Systematic review: effect of alcohol intake on adherence to outpatient medication regimens for chronic diseases. J Stud Alcohol Drugs. 2012;73(6):899-910.

8. Heaton PC, Tundia NL, Luder HR. U.S. emergency departments visits resulting from poor medication adherence: 2005-07. J Am Pharm Assoc. 2013;53(5):513-19.

9. Sokol MC, McGuigan KA, Verbrugge RR, Epstein RS. Impact of medication adherence on hospitalization risk and healthcare cost. Med Care. 2005;43(6):521-30.

10. Butler RJ, Davis TK, Johnson WG, Gardner HH. Effects of nonadherence with prescription drugs among older adults. Am J Manag Care. 2011;17(2):153-60.
11. Iuga AO, McGuire MJ. Adherence and health care costs. Risk Manag Healthc Policy. 2014;7:35-44.

12. Bardel A, Wallander MA, Svärdsudd K. Factors associated with adherence to drug therapy: a population-based study. Eur J Clin Pharmacol. 2007;63(3):307-14.

13. Brown MT, Bussell JK. Medication adherence: WHO cares? Mayo Clin Proc. 2011;86(4):304-14.

14. Welty TE, Willis SL, Welty EA. Effect of limited transportation on medication adherence in patients with epilepsy. J Am Pharm Assoc (2003). 2010;50(6):698-703.

15. Wroth TH, Pathman DE. Primary medication adherence in a rural population: the role of the patient-physician relationship and satisfaction with care. J Am Board Fam Med. 2006;19(5):478-86.

16. Kaiser Family Foundation. Hospital adjusted expenses per inpatient day. [Table time frame 2013]. 2015. Available at: http://kff.org/other/state-indicator/expenses-per-inpatient-day/. Accessed July 25, 2016.

17. Caldwell N, Srebotnjak T, Wang T, Hsia R. "How much will I get charged for this?" Patient charges for top ten diagnoses in the emergency department. PLoS One. 2013;8(2):e55491.

18. D'Hoore W, Bouckaert A, Tilquin C. Practical considerations on the use of the Charlson comorbidity index with administrative data bases. J Clin Epidemiol. 1996;49(12):1429-33.

19. University of California, San Diego. Chronic Illness and Disability Payment System. 2012. Available at: http://cdps.ucsd.edu/. Accessed July 25, 2016

20. Boswell KQ, Cook CL, Burch SP, Eaddy MT, Cantrell R. Associating medication adherence with improved outcomes: a systematic literature review. Am J Pharm Benefits. 2012;4:e97-e108. 